EXTENDED REPORT

\title{
Expression of matrix metalloproteinases in patients with Wegener's granulomatosis
}

\author{
V Bjerkeli, B Halvorsen, J K Damås, I Nordøy, A Yndestad, P Aukrust, S S Frøland
}

Ann Rheum Dis 2004;63:1659-1663. doi: 10.1136/ard.2003.017954

See end of article for authors' affiliations .......

Correspondence to: Chief Engineer V Bjerkeli, Research Institute for Internal Medicine, Medical Department, Rikshospitalet, N-0027 Oslo, Norway; vigdis.bjerkeli@ klinmed.uio.no

Accepted

16 February 2004
Background: Enhanced activity of matrix metalloproteinases (MMPs) has been reported to have a pathogenic role in several diseases such as cancer and cardiovascular disorders, and seems also to play a part in certain autoimmune diseases.

Objective: To examine whether enhanced MMP activity may also have a role in the pathogenesis of Wegener's granulomatosis (WG).

Methods: In a study group of 15 patients with WG and 15 controls, plasma levels and gene expression were measured in freshly isolated peripheral blood mononuclear cells (PBMCs) of several MMPs and their endogenous inhibitors (that is, tissue inhibitors of metalloproteinases (TIMPs)) by enzyme immunoassays and RNase protection assay, respectively.

Results: Whereas patients with WG in remission had enhanced gene expression of several MMPs and TIMPs in PBMCs, those with active disease had a selective up regulation of MMP-2 and MMP-8 compared with healthy controls, and a down regulation of TIMP-1 and TIMP-3 compared with other patients with WG. Moreover, plasma levels of TIMP-1 and MMP-8 correlated significantly with $C$ reactive protein levels, further supporting an association between activation of the MMP/TIMP system and disease activity in WG. Finally, these changes in MMP/TIMP expression in WG were accompanied by increased total MMP activity in PBMC supernatants, particularly in those with active disease, suggesting a matrix degrading net effect.

Conclusion: These findings suggest that disturbed MMP and TIMP activity has a role in the pathogenesis of WG.
W egener's granulomatosis (WG) is a necrotising vasculitis of small and medium sized vessels characterised by chronic granulomatous inflammation of the upper and lower respiratory tract, often also affecting other organs, particularly the kidneys. ${ }^{1-3}$ The pathogenesis of WG has not been fully clarified, but seems to involve enhanced leucocyte and endothelial cell activation as well as increased oxidative stress. ${ }^{3}$ Moreover, patients with WG are characterised by raised levels of inflammatory cytokines, particularly Thl related cytokines (for example, interferon $\gamma$ and interleukin 12) and tumor necrosis factor $\alpha$, possibly promoting inflammation and tissue destruction. ${ }^{56}$

Matrix metalloproteinases (MMPs) are a family of endopeptidases containing zinc with common functional domains and mechanisms of action, discovered because of their ability to degrade extracellular matrix (ECM) components. ${ }^{7}$ Although these enzymes have a key role in many physiological processes (for example, embryonic development, angiogenesis, and tissue homoeostasis and remodelling), a dysbalance between MMPs and their endogenous inhibitors (that is, tissue inhibitors of metalloproteinases (TIMPs)) may be harmful. Thus, increased MMP activity has been reported to have a pathogenic role in cancer and cardiovascular disorders ${ }^{78}$ as well as in certain autoimmune diseases-for example, systemic lupus erythematosus and rheumatoid arthritis. ${ }^{9-11}$

The immune mediated tissue damage characterising WG could potentially also involve MMP activation. At present, however, there are no data on the involvement of MMPs or TIMPs in this disorder. To elucidate this issue we examined MMP and TIMP levels in WG by different experimental approaches, trying to relate their concentrations to disease activity.

\section{PATIENTS AND METHODS \\ Patients and controls}

A total of 15 patients, seven men and eight women, with generalised WG were included in the study (table 1). All patients fulfilled the American College of Rheumatology 1990 classification criteria and the Chapel Hill Consensus Conference on the Nomenclature of Systemic Vasculitis 1992 definition for WG. ${ }^{12}{ }^{13}$ Twelve (80\%) of the patients had biopsy confirmed WG with necrotising vasculitis, granulomatous inflammation, or both, in one or more organ systems. In the three patients in whom WG could not be confirmed histologically, the diagnosis was made on the basis of the typical history, characteristic clinical findings, and a positive classic antineutrophil cytoplasmic antibody test with specificity for proteinase-3. Patients with WG were classified as having active disease or as being in remission based on clinical judgment, the Birmingham Vasculitis Activity Score (BVAS), ${ }^{13}$ as well as levels of $\mathrm{C}$ reactive protein (CRP) and the erythrocyte sedimentation rate (ESR). For comparison, blood samples were also obtained from 15 healthy controls matched for age and sex. Informed consent for blood sampling was obtained from all subjects. The study was conducted following the ethical guidelines at our hospital according to the Helsinki declaration and was approved by the hospital's authorised representative.

Abbreviations: BVAS, Birmingham Vasculitis Activity Score; CRP, C reactive protein; $E C M$, extracellular matrix; EIA, enzyme immunoassay; ESR, erythrocyte sedimentation rate; MMP, matrix metalloproteinase; PBMC, peripheral blood mononuclear cell; RPA, RNase protection assay; TIMP, tissue inhibitor of matrix metalloproteinase; WG, Wegener's granulomatosis 
Table 1 Characteristics of the patients with WG

\begin{tabular}{|c|c|c|c|c|c|c|}
\hline Patient No & Sex & $\begin{array}{l}\text { Age } \\
\text { (years) }\end{array}$ & Disease activity & $\begin{array}{l}\text { C reactive protein } \\
\text { (mg/l) }\end{array}$ & $\begin{array}{l}\text { ESR } \\
(\mathrm{mm} / 1 \mathrm{st} \mathrm{h})\end{array}$ & Drugs \\
\hline 1 & Male & 27 & Remission & 8 & 7 & $\mathrm{IVlg}$ \\
\hline 2 & Female & 65 & Remission & $<5$ & 9 & Pred $10 \mathrm{mg} \mathrm{qd}$ \\
\hline 3 & Male & 41 & Remission & 10 & 21 & Pred $10 \mathrm{mg}$ qd \\
\hline 4 & Male & 33 & Remission & $<5$ & 18 & Pred $5 \mathrm{mg}$ qd \\
\hline 5 & Female & 31 & Remission & $<5$ & 10 & TMP/SMX \\
\hline 6 & Female & 52 & Remission & 5 & 8 & - \\
\hline 7 & Male & 59 & Remission & 5 & 17 & $\mathrm{TMP} / \mathrm{SMX}$ \\
\hline 8 & Female & 37 & Remission & $<5$ & 11 & Pred $10 \mathrm{mg}$ qd \\
\hline 9 & Female & 35 & Active & 14 & 110 & Pred $20 \mathrm{mg}$ bid, CYC \\
\hline 10 & Male & 28 & Active & 29 & 61 & Pred $15 \mathrm{mg}$ qd, CYC \\
\hline 11 & Male & 58 & Active & 161 & 100 & Pred $15 \mathrm{mg} \mathrm{qd}$, etan \\
\hline 12 & Female & 84 & Active & 45 & 53 & Pred $20 \mathrm{mg}$ bid, CYC \\
\hline 13 & Female & 56 & Active & 62 & 96 & Pred $20 \mathrm{mg}$ qd, CYC \\
\hline 14 & Female & 35 & Active & 57 & 30 & Pred $20 \mathrm{mg}$ bid, IVlg \\
\hline 15 & Male & 58 & Active & 16 & 47 & Pred $20 \mathrm{mg}$ qd, CYC \\
\hline
\end{tabular}

ESR, erythrocyte sedimentation rate; Pred, prednisolone; IVlg, intravenous immunoglobulin; TMP/SMX, trimetroprim/sulfamethoxazole; CYC, cyclophosphamide; etan, etanercept; bid, twice a day; qd, every day.

\section{Blood sampling protocol}

Peripheral venous blood was drawn into pyrogen-free tubes without additives (serum) or with EDTA as anticoagulant (plasma). The tubes were immediately immersed in melting ice and centrifuged ( $1500 \mathrm{~g}$ for 10 minutes) within $20 \mathrm{~min}$ utes (plasma) or allowed to clot before centrifugation (serum). All samples were stored at $-80^{\circ} \mathrm{C}$ and thawed only once.

\section{Isolation and culturing of cells}

Peripheral blood mononuclear cells (PBMCs) were obtained from heparinised blood by Isopaque-Ficoll (Lymphoprep, Nycomed Pharma, Oslo, Norway) gradient centrifugation within 45 minutes. For mRNA analyses (see below), cell pellets were stored in liquid nitrogen until used for RNA extraction. For analyses of MMP activity, PBMCs $\left(2 \times 10^{6} / \mathrm{ml}\right.$, $1 \mathrm{ml} /$ well) were incubated in flat bottomed, 24 well microtitre plates (Costar, Cambridge, MA) with serum-free medium (X-vivo; Bio-Whittaker, Walkersville, MD). After different times, cell-free supernatants were removed and stored at $-80^{\circ} \mathrm{C}$.

\section{RNase protection assay (RPA)}

Total RNA was extracted from PBMCs using RNeasy columns (Qiagen, Hilden, Germany) and stored in RNA storage solution (Ambion, Austin, TX) at $-80^{\circ} \mathrm{C}$. RPA was performed as previously described using the hMMP-2 multiprobe (Pharmingen, San Diego, CA). ${ }^{14}$ The mRNA signal was normalised to the signal from the housekeeping gene ribosomal protein L32 (rpL32).

\section{Zymography}

Gelatinase activity was detected in PBMC supernatant after incubation for 18 hours, as previously described. ${ }^{15}$ Briefly, $46 \mu \mathrm{g}$ of protein was incubated with a sample buffer containing $2 \%$ sodium dodecyl sulphate. Human pro-MMP9 and active MMP-9 (Oncogene, Cambridge, MA) were run in a separate lane to ensure identification of MMP activity. Gels were scanned by Kodak 440 CF imaging station (Vector, Burlingame, CA), and the software Total Laboratory (Phoretix, Newcastle, UK) was used for quantification.

\section{Total MMP activity}

Total MMP activity in PBMC supernatants was measured by a fluorogenic peptide substrate (R\&D systems, Minneapolis, $\mathrm{MN}$; cat no: ES001) using the protocol recommended by the manufacturer. Briefly, the MMP substrate was diluted in TCN
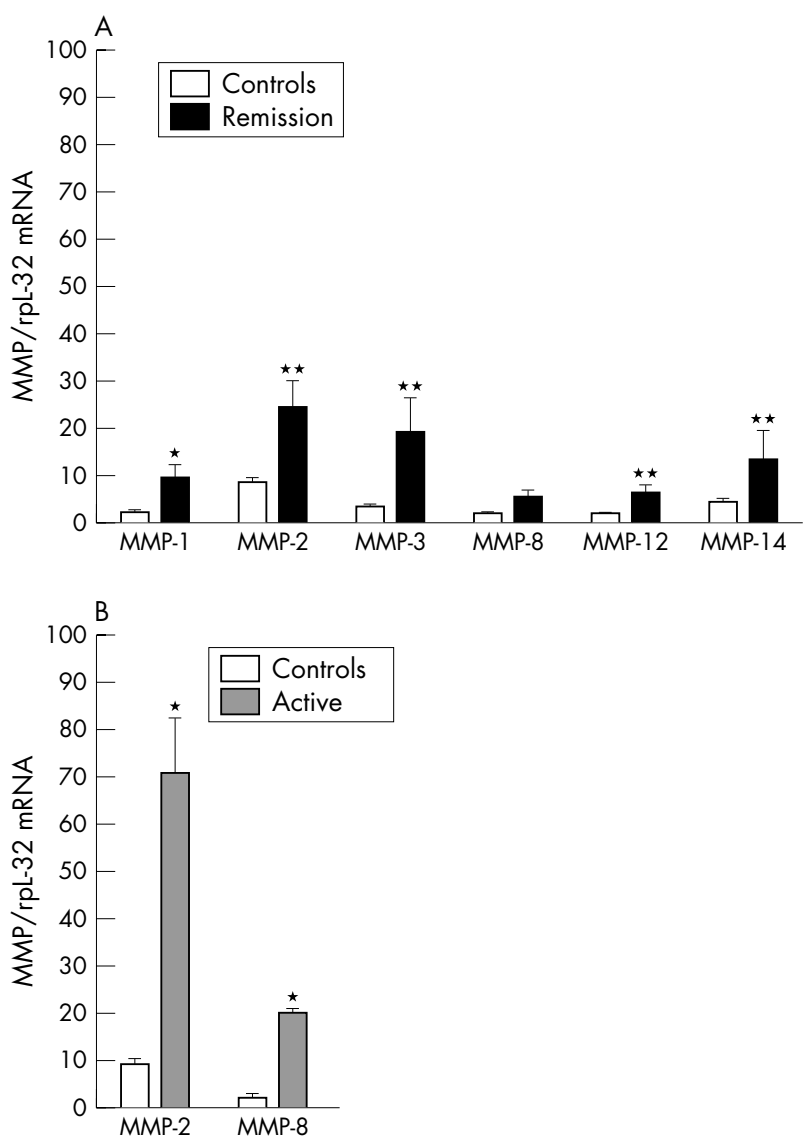

Figure 1 Gene expression (RPA) of MMPs in relation to the control gene rpL32 in PBMCs from patients with WG classified as being in remission $(n=8)(A)$ or as having active disease $(n=7)(B)$ according to the BVAS, serum levels of CRP, and ESR (see table 1) and 15 healthy controls. Data are given as mean (SEM). ${ }^{*} p<0.01$ and ${ }^{* *} p<0.001$ versus healthy controls.

buffer $(50 \mathrm{mM}$ Tris $\mathrm{HCl}, 150 \mathrm{mM} \mathrm{NaCl}, 10 \mathrm{mM} \mathrm{CaCl}$; $\mathrm{pH} 7.5$ ) and added to the supernatants (preactivated by aminophenylmercuric acetate (Sigma, St Louis, MO) for 1 hour) before incubation at $37^{\circ} \mathrm{C}$. After 30 minutes the total MMP activity was determined on a fluorimeter (FLX 800 Microplate Fluorescence Reader; Bio-Tek Instruments, Winooski, VT). 


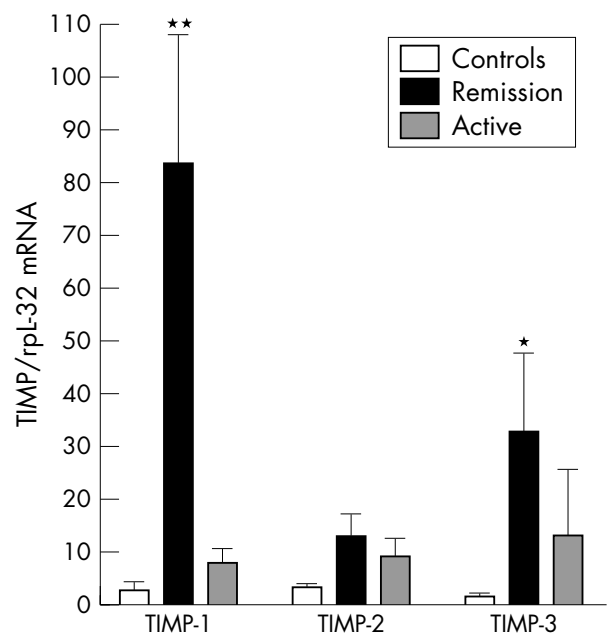

Figure 2 Gene expression (RPA) of TIMPs in relation to the control gene rpL32 in PBMCs from 15 patients with WG classified as having active disease $(n=7)$ or being in remission $(n=8)$ according to the BVAS, serum levels of CRP, and ESR (see table 1) and 15 healthy controls. Data are given as mean (SEM). ${ }^{*} p<0.01$ and ${ }^{* *} p<0.001$ versus active disease and healthy controls.

\section{Enzyme immunoassays (EIAs)}

Plasma concentrations of MMP-3, MMP-8, and TIMP-1 were quantified by EIAs (R\&D System). MMP-2 levels were analysed by EIA (Amersham Biosciences, Buckinghamshire, UK). The intra- and interassay coefficients of variation were $<10 \%$ for all EIAs.

\section{Statistical analysis}

Statistical comparisons between patients with WG and healthy controls and between different groups of patients with WG were performed using the Mann-Whitney rank sum test. Relationships between variables were tested using Spearman's rank correlation test. Values of $\mathrm{p}$ were two-sided and were considered significant when $<0.05$.

\section{RESULTS}

\section{Gene expression levels of MMPs and TIMPs}

We first analysed the gene expression of several MMPs and TIMPs in PBMCs from patients with WG $(\mathrm{n}=15)$ and healthy controls $(n=15)$. Figure 1 shows that while several MMPs (that is, MMP-1, MMP-2, MMP-3, MMP-12, and MMP-14) were moderately up regulated in patients with WG in remission, those with active disease showed a different pattern, with a selective and marked up regulation of MMP-2 $(p<0.01)$ and MMP-8 $(p<0.01)$ compared with gene expres-
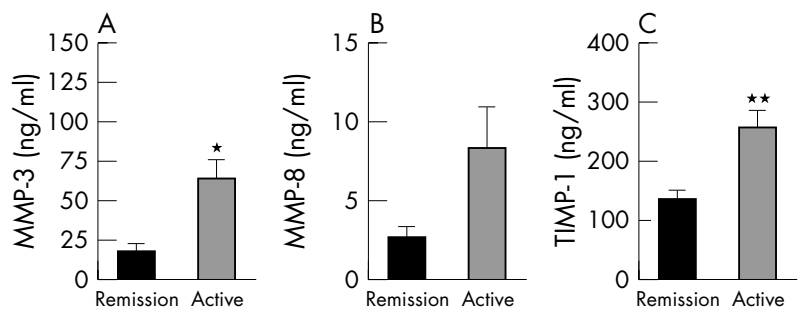

Figure 4 Plasma levels of MMP-3 (A), MMP-8 (B), and TIMP-1 (C) in 15 patients with WG classified as having active disease $(n=7)$ or being in remission $(n=8)$ according to the BVAS, serum levels of CRP, and ESR (see table 1). Data are given as mean (SEM). ${ }^{*} p<0.01$ and ${ }^{* *} p<0.005$ versus patients in remission.

sion in healthy controls. Furthermore, whereas patients with WG in remission had increased expression of TIMP-1 and TIMP-3 compared with healthy controls, such an up regulation was not seen in patients with active disease. In fact, TIMP-1 and TIMP-3 were significantly down regulated in those with active disease compared with other patients with WG (fig 2).

\section{Plasma levels of MMPs and TIMPs}

To further examine the MMP/TIMP system in WG, we examined plasma levels of MMP-2, MMP-3, MMP-8, and TIMP-1, all shown to be dysregulated in WG at the mRNA levels (see above), in 15 patients with WG and 15 healthy controls. Figure 3 shows that patients with WG had enhanced plasma levels of MMP-2, MMP-3, and TIMP-1 compared with healthy controls. Moreover, when the patients with WG were classified as having active disease $(n=7)$ or being in remission $(n=8)$ (table 1$)$, we found that those with active disease had raised plasma levels of MMP-3, MMP-8, and TIMP- 1 compared with the other patients with WG, although the difference for MMP-8 $(p=0.053)$ did not reach significance (fig 4). Moreover, plasma levels of TIMP-1 and MMP-8, but not MMP-2 and MMP-3, correlated significantly with CRP levels in the patient group as a whole (fig 5).

\section{MMP activity}

To examine if this altered MMP and TIMP expression resulted in any changes in MMP activity, we first measured total MMP activity in PBMC supernatants from nine patients with WG (five in remission and four with active disease) and eight healthy controls. Figure 6 shows that the substrate used in this assay can be cleaved by MMP-1, MMP-2, MMP-7, MMP-8, MMP-9, MMP-12, and MMP-13. The changes in MMP and TIMP expression in WG were accompanied by a significant increase in total MMP activity, particularly in
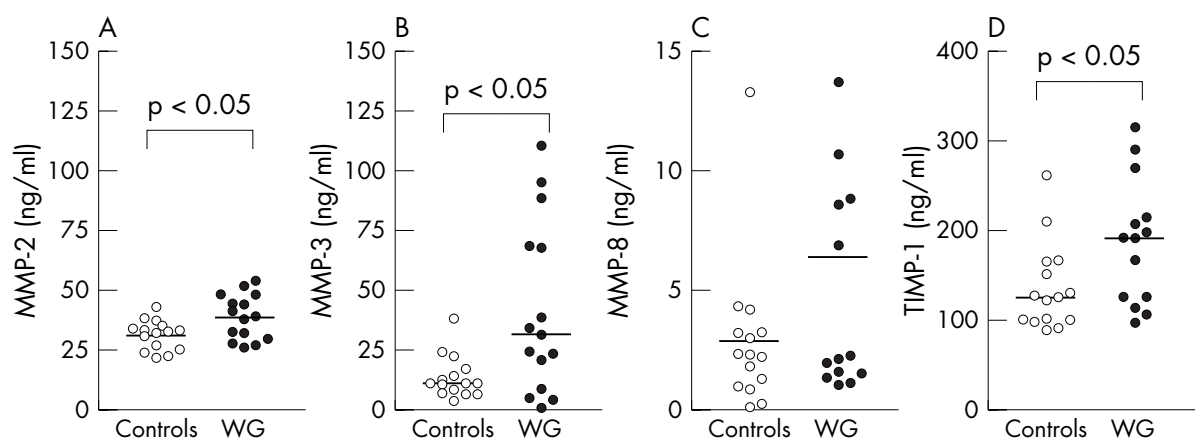

Figure 3 Plasma levels of MMP-2 (A), MMP-3 (B), MMP-8 (C), and TIMP-1 (D) in 15 patients with WG and 15 healthy controls. Horizontal lines represent median values. 

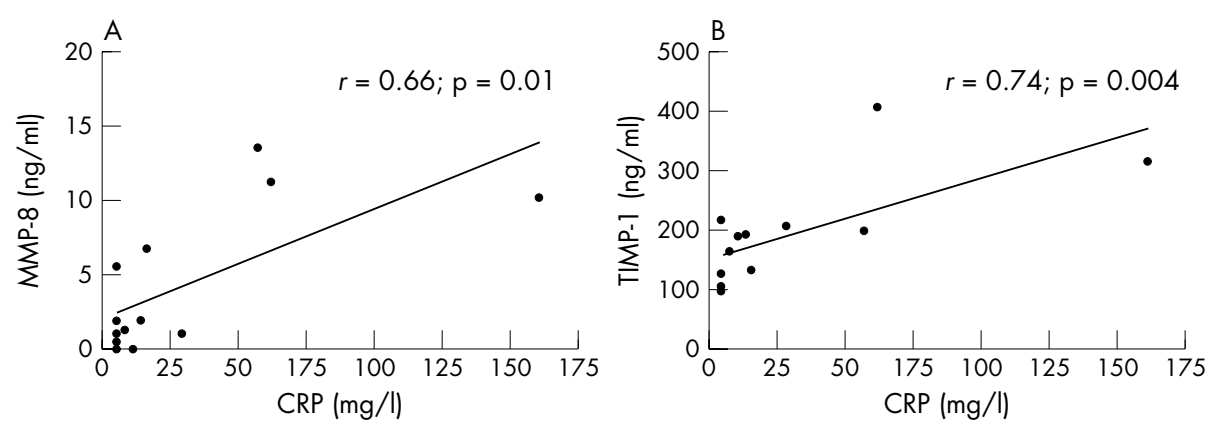

Figure 5 Correlation between plasma levels of CRP and MMP-8 (A) and TIMP-1 (B) in 15 patients with WG.

those with active disease (data not shown), suggesting a matrix degrading net effect. Moreover, because MMP-9 was not included in the RPA probe, we analysed MMP-9 activity by zymography in PBMC supernatants from six patients with WG and six healthy controls. Comparable to total MMP activity, patients with WG had increased MMP-9 activity both for the pro-enzyme $(94 \mathrm{kDa})$ and for its active form (84 kDa) (fig 6).

\section{DISCUSSION}

In this study we report that patients with WG are characterised by marked alterations in gene expression of several MMPs and TIMPs in PBMCs. Whereas patients with WG in remission had moderately enhanced expression of several MMPs and TIMPs, those with active disease had a selective and marked up regulation of MMP-2 and MMP-8 compared with healthy controls, and a down regulation of TIMP-1 and TIMP-3 compared with other patients with WG. Finally, these changes in MMP/TIMP expression in WG were accompanied by increased total MMP activity in PBMC supernatants, particularly in those with active disease, indicating a matrix degrading net effect. Although we cannot exclude the possibility that some of these findings may reflect the use of immunosuppressive drugs in the patient group, our

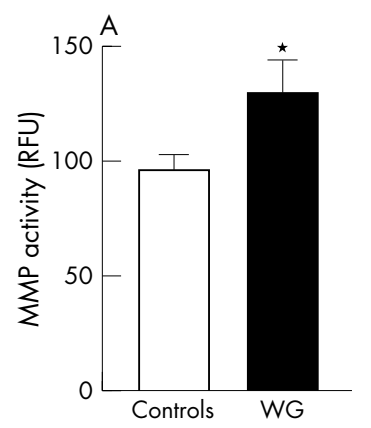

B

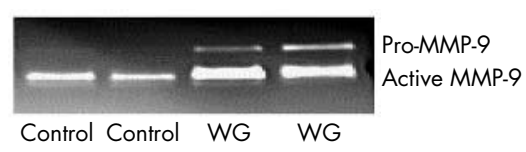

Figure 6 MMP activity in PBMC supernatants from patients with WG and healthy controls. (A) Total MMP activity in nine patients with WG and eight healthy controls using a fluorogenic peptide substrate (see "Patients and methods"). (B) Gelatinolytic (MMP-9) activity in PBMC supernatants after incubation for 18 hours in two representative patients with WG and two healthy controls. Gelatinolytic activity was seen as lytic bands and the majority of these were identified as pro-MMP-9 and active MMP-9. Note: pro-MMP-9 was only detected in the WG group. $\mathrm{RFU}$, relative fluorescence units. results suggest a role for disturbed MMP/TIMP activity in the pathogenesis of WG.

MMPs are a pivotal family of zinc enzymes responsible for degradation of the ECM components. ${ }^{7}$ In this study we show that PBMCs from patients with WG have markedly enhanced expression of several MMPs with the potential to induce degradation of tissue components such as collagen, fibronectin, and various proteoglycans. ${ }^{78}$ Notably, patients with WG with active disease were characterised by a selective and marked up regulation of MMP-2 and MMP- 8 and, as for MMP-8, such an association with disease activity was also found for plasma levels. Among collagenases, MMP-8 most effectively hydrolyses the native type I and II collagens. ${ }^{16}$ It has been suggested that this MMP has a pathogenic role in disorders such as chronic bronchiectasis, cystic fibrosis, and rheumatoid arthritis, ${ }^{7}{ }^{17}$ and although we have no data on MMP-8 enzymatic activity, our results suggest that this MMP also may be play a part in the pathogenesis of WG.

The activity of MMPs is regulated by several factors such as the levels of their naturally occurring inhibitors (for example, TIMPs). ${ }^{78}$ Although the increase in MMP levels was accompanied by enhanced expression of TIMP-1 and TIMP3 in patients with WG in remission, this was not found in those with active disease. Such an imbalance between MMPs and their endogenous inhibitors was also indicated at the functional level, showing enhanced total MMP activity in patients with WG, particularly in those with active disease. While patients with active disease showed no increase in TIMP-1 gene expression in PBMCs, these patients had raised plasma levels of this inhibitor. The reasons for these apparently discrepant results are at present not clear but several possibilities exist. Firstly, plasma levels of TIMP-1 will not only reflect the production in PBMCs but also other cells, such as endothelial cells and tissue macrophages, may be of even more importance. Moreover, while the TIMP-1 level in PBMCs reflects the actual TIMP-1 expression in this cell population, plasma levels of TIMP-1 may also be regarded as a measure of the activity in the TIMP/MMP system..$^{7818}$

Several studies have shown potent induction of MMP expression by inflammatory cytokines such as tumour necrosis factor $\alpha$, interleukin 12, and chemokines. ${ }^{7819} 20$ Because a number of reports have shown increased levels of such inflammatory cytokines in $\mathrm{WG}^{5}{ }^{621}$ it is not inconceivable that cytokine induced MMP activity may also be operating in this disorder. Whatever the mechanisms, this disturbed MMP/TIMP expression in WG with net ECM degrading effects may have several pathogenic consequences. Thus, studies in animal models suggest that enhanced MMP activity may promote tissue destruction, remodelling, and fibrosis in the lungs. ${ }^{17} 2223$ Moreover, recent studies in rat models suggest the involvement of MMP-2 and MMP-14 in the pathogenesis of crescentic glomerulonephritis. ${ }^{24}$ Furthermore, although the role of MMPs in various nasal 
disorders is largely unclear, enhanced MMP activity seems to play a part in the pathogenesis of nasal polyps and allergic rhinitis, ${ }^{25} 26$ and even more importantly with relevance to WG, several MMPs (for example, MMP-1, MMP-3, and MMP-8) have been shown to promote degradation of type II collagen and proteoglycan in nasal cartilages. ${ }^{27-29}$ Finally, while inflammatory cytokines are potent inducers of MMPs, enhanced MMP activity may itself promote inflammation and granuloma formation, representing a possible link between tissue destruction/remodelling and inflammation in various disorders. ${ }^{30}$

Unbalanced MMP activity has been demonstrated in several disorders, such as arthritis, malignancies, and arteriosclerosis, and although not specific for WG, our demonstration of a correlation between MMP/TIMP levels and disease activity, as assessed by both clinical and laboratory criteria, suggests that such a pathogenic link may also be operating in WG. Although these findings will have to be confirmed also at the tissue level, particularly in target organs, our results suggest that WG may be added to this list of disorders characterised by dysregulated MMP/ TIMP levels, possibly representing a new target for treatment in this disorder.

\section{ACKNOWLEDGEMENTS}

This work was supported by the Research Council of Norway, the Norwegian Cancer Society, and Medinnova Foundation.

We thank Ellen Lund Sagen, Bodil Lunden, and Azita Rashidi for excellent technical assistance.

\section{Authors' affiliations}

V Bjerkeli, B Halvorsen, J K Damås, A Yndestad, P Aukrust, S S Frøland, Research Institute for Internal Medicine, Medical Department, Rikshospitalet University Hospital, N-0027 Oslo, Norway

I Nordøy, P Aukrust, S S Frøland, Section of Clinical Immunology and Infectious Diseases, Medical Department, Rikshospitalet University Hospital, N-0027 Oslo, Norway

\section{REFERENCES}

1 Fauci A, Wolff S. Wegener's granulomatosis. Medicine (Baltimore) 1973;73:535-61.

2 Jennette JC, Falk RJ, Andrassy K. Nomenclature of systemic vasculitides: proposal of an international consensus conference. Arthritis Rheum 1994;37:187-92.

3 Yi ES, Colby TV. Wegener's granulomatosis. Semin Diagn Pathol 2000;18:34-46.

4 Schnabel A, Csernok E, Braun J, Gross WL. Activation of neutrophils, eosinophils, and lymphocytes in the lower respiratory tract in Wegener's granulomatosis. Am J Respir Crit Care Med 2000;161:399-405.

5 Lamprecht $\mathbf{P}$, Kumanovics G, Mueller A, Csernok E, Komocsi A, Trabandt A, et al. Elevated monocytic IL-12 and TNF- $\alpha$ production in Wegener's granulomatosis is normalized by cyclophosphamide and corticosteroid therapy. Clin Exp Immunol 2002; 128:181-6.

6 Komocsi A, Lamprecht P, Csernok E, Mueller A, Holl-Ulrich K, Seitcer U, et al. Peripheral blood and granuloma CD4(+)CD28(-) T cells are major source of interferon-gamma and tumor necrosis factor alpha in Wegener's granulomatosis. Am J Pathol 2002;160:1717-24.

7 Matrisian LM. Metalloproteinases and their inhibitors in matrix remodelling. Trends Genet 1990;6:121-5.
8 Visse R, Nagase H. Matrix metalloproteinases and tissue inhibitors of metalloproteinases: structure, function, and biochemistry. Circ Res 2003;92:827-39

9 Yoshihara Y, Nakamura H, Obata K, Yamada H, Hayakawa T, Fujikawa K, et al. Matrix metalloproteinases and tissue inhibitors of metalloproteinases in synovial fluids from patients with rheumatoid arthritis or osteoarthritis. Ann Rheum Dis 2000;59:455-61.

10 Faber-Elmann A, Sthoeger Z, Tcherniack A, Dayman M, Mozes E. Activity of matrix metalloproteinase-9 is elevated in sera of patients with systemic lupus erythematosus. Clin Exp Immunol 2002;127:393-8.

11 Mengshol JA, Mix KS, Brinckerhoff CE. Matrix metalloproteinases as therapeutic targets in arthritic diseases. Arthritis Rheum 2002;46:13-20.

12 Leavitt RY, Fauci AS, Bloch DA. The American College of Rheumatology 1990 criteria for the classification of Wegener's granulomatosis. Arthritis Rheum 1990;33:1 101-7.

13 Luqmani RA, Bacon PA, Moots RJ, Janssen BA, Pall A, Emery P, et al. Birmingham Vasculitis Activity Score (BVAS) in systemic necrotizing vasculitis. QJM 1994;87:671-8.

14 Damås JK, Eiken HG, Øie E, Bjerkeli V, Yndestad Y, Ueland T, et al. Myocardial expression of CC- and CXC-chemokines and their receptors in human end-stage heart failure. Cardiovasc Res 2000;47:778-87.

15 Wæhre T, Halvoren B, Damås JK, Yndestad A, Brosstad F, Gullestad L, et al. Inflammatory imbalance between IL-10 and TNF- $\alpha$ in unstable anginapotential plaque stabilizing effect of IL-10. Eur J Clin Invest 2002;32:803-10.

16 Hanemaaijer R, Sorsa T, Konttinen YT, Ding Y, Sutinen M, Visser H, et al. Matrix metalloproteinase- 8 is expressed in rheumatoid synovial fibroblasts and endothelial cells. Regulation by tumor necrosis factor- $\alpha$ and doxycycline. J Biol Chem 1997;272:31504-9.

17 Power C, O'Connor CM, MacFarlane D, O'Mahoney S, Gaffney K, Hayes J, et al. Neutrophil collagenase in sputum from patients with cystic fibrosis. Am J Respir Crit Care Med 1994;150:818-22.

18 Wiercinska-Drapalo A, Jaroszewicz J, Flisiak R, Prokopowicz D. Plasma matrix metalloproteinase- 1 and tissue inhibitors of metalloproteinase- 1 as biomarkers of ulcrative colitis activity. World J Gastroenterol 2003;9:2843-5.

19 Abraham M, Shapiro S, Lahat N, Miller A. The role of IL-18 and IL-12 in the modulation of matrix metalloproteinases and their tissue inhibitors in monocytic cells. Int Immunol 2002;14:1440-57.

20 Siwik DA, Chang DL, Colucci WS. Interleukin-1 $\beta$ and tumor necrosis factor- $\alpha$ decrease collagen synthesis and increase matrix metalloproteinase activity in cardiac fibroblasts in vitro. Circ Res 2000;86:1259-65

21 Muller Kobold AC, Kallenberg CG, Tervaert JW. Monocyte activation in patients with Wegener's granulomatosis. Ann Rheum Dis 1999;58:237-45.

22 Atkinson JJ, Senior RM. Matrix metalloproteinase-9 in lung remodelling. Am J Respir Cell Mol Biol 2003;8:12-24.

23 Ohbayashi H. Matrix metalloproteinases in lung diseases. Curr Protein Pept Sci 2002;3:409-21.

24 Hayashi K, Horikoshi S, Osada S, Shofuda K, Shirato I, Tomino Y Macrophage-derived MT1-MMP and increased MMP-2 activity are associated with glomerular damage in crescentic glomerulonephritis. J Pathol 2002;191:299-305.

25 Lechapt-Zalcman E, Coste A, d'Ortho MP, Frisdal E, Harf A, Lafu C, et al. Increased expression of matrix metalloproteinase-9 in nasal polyps. J Pathol 2001;193:233-41.

26 Shaida A, Kenyon G, Devalia J, Davies RJ, MacDonald TT, Pender SLE. Matrix metalloproteinases and their inhibitors in the nasal mucosa of patients with perennial allergic rhinitis. J Allergy Clin Immunol 2001;108:791-6.

27 Hui W, Rowan AD Cawston T. Insulin-like growth factor 1 blocks collagen release and down regulates matrix metalloproteinase- $-1,-3,-8$, and -13 mRNA expression in bovine nasal cartilage stimulated with oncostatin in combination with interleukin 1 $\alpha$. Ann Rheum Dis 2001;60:254-61.

28 Billinghurst RC, Wu W, lonescu M, Reiner A, Dahlberg L, Chen J, et al. Comparison of the degradation of type II collagen and proteoglycan in nasal and articular cartilages induced by interleukin-1 and the selective inhibition of type II collagen cleavage by collagenase. Arthritis Rheum 2000;43:664-72

29 Bottomley KM, Borkakoti N, Bradshaw D, Brown PA, Broadhurst J, Brudd JM, et al. Inhibition of bovine nasal cartilage degradation by selective matrix metalloproteinase inhibitors. Biochem J 1997;323:483-8.

30 McCawley L, Matrisian LM. Matrix metalloproteinases: they're not just for matrix anymore. Curr Opin Cell Biol 2001;13:534-40. 